\title{
Single Nucleotide Polymorphisms Associated with Coronary Heart Disease Predict Incident Ischemic Stroke in the Atherosclerosis Risk in Communities Study
}

\author{
Alanna C. Morrison ${ }^{a}$ Lance A. Bare ${ }^{d}$ May M. Luke ${ }^{d}$ James S. Pankow ${ }^{e}$ \\ Thomas H. Mosley ${ }^{f}$ James J. Devlin ${ }^{d}$ James T. Willerson ${ }^{c}$ Eric Boerwinkle ${ }^{a} b$ \\ ${ }^{a}$ Human Genetics Center and Division of Epidemiology and ${ }^{b}$ Institute of Molecular Medicine, University of \\ Texas Health Science Center at Houston, and 'Texas Heart Institute, Houston, Tex., ' Celera, Alameda, Calif., \\ e Division of Epidemiology and Community Health, University of Minnesota, Minneapolis, Minn., and \\ ${ }^{f}$ Department of Medicine (Geriatrics), University of Mississippi Medical Center, Jackson, Miss., USA
}

\section{Key Words}

Genetic factor $\cdot$ Coronary heart disease $\cdot$ Ischemic stroke •

Myocardial infarction $\cdot$ Single nucleotide polymorphisms

\begin{abstract}
Ischemic stroke and coronary heart disease (CHD) may share genetic factors contributing to a common etiology. This study investigates whether 51 single nucleotide polymorphisms (SNPs) associated with CHD in multiple antecedent studies are associated with incident ischemic stroke in the Atherosclerosis Risk in Communities (ARIC) study. From the multiethnic ARIC cohort of 14,215 individuals, 495 validated ischemic strokes were identified. Cox proportional hazards models, adjusted for age and gender, identified three SNPs in Whites and two SNPs in Blacks associated with incident stroke ( $p \leq 0.05$ ). The rs 11628722 polymorphism in SERPINA9 was associated with incident stroke in Whites and Blacks, even after taking into account traditional risk factors. The idea that ischemic stroke and CHD may share some common genetic factors, such as variation in SERPINA9, should be investigated in other studies.

Copyright $\odot 2008$ S. Karger AG, Basel
\end{abstract}

In the United States, cardiovascular disease is the leading cause of death [1]. Coronary heart disease (CHD) and stroke, respectively, represent the first and third most common causes of mortality and morbidity [1]. Although these diseases involve different vascular beds, at least a portion of $\mathrm{CHD}$ and ischemic stroke cases are known to be the result of atherosclerotic disease and its complications [2]. The idea that stroke and CHD share some predisposing pathologies is supported by the fact that these diseases share risk factors, such as age, gender, smoking, diabetes and hypertension [2]. These and other traditional risk factors have been incorporated into risk prediction models for CHD and stroke [3, 4]. Shared risk factors for these diseases may also encompass genetic factors, such as gene variants involved in atherosclerosis. Recently, risk prediction models for CHD have been improved by incorporating a genetic risk score that aggregates the contribution of multiple single nucleotide polymorphisms (SNPs) [5]. Given the shared atherosclerotic pathology between CHD and stroke and the possible involvement of common genetic risk factors, 51 putative functional SNPs that had been found to be associated

\section{KARGER}

(C) 2008 S. Karger AG, Basel

Fax +41613061234 E-Mail karger@karger.ch www.karger.com www.karger.com/ced
Alanna Morrison, $\mathrm{PhD}$

Human Genetics Center, University of Texas Health Science Center at Houston 1200 Herman Pressler, Suite 453E

Houston, TX 77030 (USA)

Tel. +1 713500 9800, Fax +1 713500 0900, E-Mail Alanna.C.Morrison@uth.tmc.edu 
with CHD in multiple studies $[5,6]$ were evaluated for their ability to predict incident ischemic stroke in the Atherosclerosis Risk in Communities (ARIC) study. These SNPs were further investigated for their ability to predict ischemic stroke independent of established risk factors.

\section{Subjects and Methods}

\section{The ARIC Study}

Study participants were selected from the ARIC study, a prospective investigation of atherosclerosis and its clinical sequelae involving 15,792 individuals aged 45-64 years at recruitment (1986-1989). Subjects were selected by probability sampling from four communities: Forsyth County, N.C.; Jackson, Miss. (Blacks only); northwestern suburbs of Minneapolis, Minn., and Washington County, Md., USA. The initial clinical exams included a home interview to ascertain cardiovascular risk factors, socioeconomic factors and family medical history, clinical examination and blood drawing for laboratory determinations. A detailed description of the ARIC study design and methods has been published elsewhere [7].

\section{Incident Ischemic Stroke}

Ischemic stroke was determined by contacting participants annually to identify hospitalizations during the previous year, and by surveying discharge lists from local hospitals and death certificates from state vital statistics offices for potential cerebrovascular events $[7,8]$. Hospital records were obtained, abstracted and classified by computer algorithm and physician review. Details on quality assurance for ascertainment and classification of ischemic stroke events have been published elsewhere [8]. Ischemic stroke events were defined as validated definite or probable hospitalized embolic $(n=87)$ or thrombotic $(n=408)$ brain infarctions. Participants were excluded from this analysis if they had a positive or unknown history of prevalent stroke; transient ischemic attack/stroke symptoms or CHD at the initial visit; ethnic background other than White or Black; a Black ethnic background but not from Jackson, Miss., USA; restrictions on use of their DNA, or missing data for any of the traditional cardiovascular or cerebrovascular risk factors. The remaining 14,215 participants were followed for incident ischemic stroke for a mean of 13.5 years and 495 incident ischemic stroke cases were identified.

\section{Examination and Laboratory Measures}

Cardiovascular risk factors considered in this study were measured at baseline and included age, gender, waist-to-hip ratio, diabetes, hypertension, and smoking status. The ratio of waist (umbilical level) and hip (maximum buttocks) circumference was calculated as a measure of fat distribution. Diabetes was defined by a fasting glucose level $\geq 126 \mathrm{mg} / \mathrm{dl}$, a nonfasting glucose level $\geq 200 \mathrm{mg} / \mathrm{dl}$, or a self-reported physician diagnosis of diabetes or use of diabetes medication. Seated blood pressure was measured three times with a random zero sphygmomanometer and the last two measurements were averaged. An interviewer-administered questionnaire was applied to assess the use of antihypertensive medications. Hypertension was defined as systolic blood pressure $\geq 140 \mathrm{~mm} \mathrm{Hg}$ or diastolic blood pressure $\geq 90 \mathrm{~mm} \mathrm{Hg}$ or current use of antihypertensive medication. Cigarette smoking status was classified as current or not current. The study protocol was approved by the Institutional Review Boards of the collaborating institutions, and informed written consent was obtained from each participant.

\section{SNP Selection and Genotype Determination}

Fifty-one putative functional SNPs associated with CHD in multiple antecedent studies, other than the ARIC study, were considered in this study. A detailed description of the antecedent studies is presented elsewhere [6]. Briefly, risk alleles for 49 SNPs were specified based on a significant association with myocardial infarction in at least two antecedent case-control studies. These studies involved myocardial infarction cases and controls recruited by either the Cleveland Clinic Foundation Heart Center, Cleveland, Ohio, or the Genomic Resource in Arteriosclerosis at the University of California, San Francisco, Calif., USA. All cases in these two studies had a history of myocardial infarction and the controls did not, and all subjects were self-described, non-Hispanic Caucasians. The risk alleles for two additional SNPs were specified based on an association with CHD in the placebo arms of two CHD prevention trials: the Cholesterol and Recurrent Events study [9] and the West of Scotland Coronary Prevention study [10]. One of these SNPs (rs20455 in KIF6) was significantly associated with CHD after correction for multiple testing [11]. The second SNP associated with CHD in the Cholesterol and Recurrent Events study and the West of Scotland Coronary Prevention study was rs11666735 in FCAR [12].

Genotyping of the 51 SNPs in the ARIC study was carried out using PCR-based amplification of genomic DNA followed by an allele-specific oligonucleotide ligation assay similar to a previously described procedure [13]. The primer sequences for genotyping these 51 SNPs are available from the authors upon request.

\section{Statistical Analyses}

Agreement of genotype frequencies with Hardy-Weinberg equilibrium expectations was tested separately in Whites and Blacks using a $\chi^{2}$ goodness-of-fit test in noncases, stratified by ethnicity. Deviation from Hardy-Weinberg equilibrium was determined by a $\mathrm{p}$ value less than 0.05 . Cox proportional hazards models were used to model time to incident ischemic stroke. The follow-up time interval was defined as the time between the initial clinical visit and the end of follow-up, which for cases was the date of the first ischemic stroke event and for noncases was December 31,2002 , the date of death, or the date of last contact if lost to follow-up. Each model was evaluated separately in Whites and Blacks and included a given SNP (modeled as the additive effect of the prespecified risk allele), age and gender. Additional risk factors evaluated as potential confounders in the Cox proportional hazards models included waist-to-hip ratio, diabetes, hypertension, and smoking status [14]. SNPs and risk factors were assessed for statistical significance in the models by the Wald statistic. A two-sided p value of 0.05 was used to assess statistical significance with ischemic stroke and no attempt was made to adjust for multiple comparisons within this study. 
Table 1. Baseline characteristics

\begin{tabular}{|c|c|c|c|c|c|c|}
\hline \multirow[t]{2}{*}{ Characteristics } & \multicolumn{3}{|c|}{ Whites $(\mathrm{n}=10,401)$} & \multicolumn{3}{|c|}{ Blacks $(\mathrm{n}=3,814)$} \\
\hline & $\begin{array}{l}\text { cases } \\
(\mathrm{n}=275)\end{array}$ & $\begin{array}{l}\text { noncases } \\
(\mathrm{n}=10,126)\end{array}$ & $\mathrm{p}$ value $^{1}$ & $\begin{array}{l}\text { cases } \\
(\mathrm{n}=220)\end{array}$ & $\begin{array}{l}\text { noncases } \\
(n=3,594)\end{array}$ & $\mathrm{p}$ value $^{1}$ \\
\hline Age $($ mean $\pm S D)$, years & $57.59 \pm 5.32$ & $54.10 \pm 5.69$ & $<0.01$ & $55.21 \pm 5.79$ & $53.25 \pm 5.79$ & $<0.01$ \\
\hline Waist-to-hip ratio (mean \pm SD) & $0.96 \pm 0.07$ & $0.92 \pm 0.08$ & $<0.01$ & $0.94 \pm 0.07$ & $0.92 \pm 0.08$ & $<0.01$ \\
\hline Male & $162(59)$ & $4,569(45)$ & $<0.01$ & $97(44)$ & $1,318(37)$ & 0.03 \\
\hline Hypertensive & $137(50)$ & $2,516(25)$ & $<0.01$ & $168(77)$ & $1,903(53)$ & $<0.01$ \\
\hline Diabetic & $55(20)$ & $799(8)$ & $<0.01$ & $94(44)$ & $596(17)$ & $<0.01$ \\
\hline Smoker & $90(33)$ & $2,455(24)$ & $<0.01$ & $81(37)$ & $1,036(29)$ & 0.01 \\
\hline
\end{tabular}

Figures in parentheses indicate percentages.

${ }^{1} \mathrm{p}$ value represents a comparison between cases and noncases within an ethnic group.

Table 2. SNPs associated with incident ischemic stroke in the ARIC study

\begin{tabular}{|c|c|c|c|c|c|c|c|c|c|c|}
\hline \multirow{2}{*}{$\begin{array}{l}\text { Gene } \\
\text { symbol }\end{array}$} & \multirow[t]{2}{*}{ SNP ID } & \multirow[t]{2}{*}{ Function $^{\mathrm{a}}$} & \multirow{2}{*}{$\begin{array}{l}\text { Risk-raising } \\
\text { allele } \\
\text { (frequency) }\end{array}$} & \multirow{2}{*}{$\begin{array}{l}\text { Risk-lowering } \\
\text { allele } \\
\text { (frequency) }\end{array}$} & \multicolumn{3}{|c|}{ Model $1^{\mathrm{b}}$} & \multicolumn{3}{|c|}{ Model $2^{c}$} \\
\hline & & & & & HRR & $95 \% \mathrm{CI}$ & $\mathrm{p}$ value & HRR & $95 \% \mathrm{CI}$ & $\mathrm{p}$ value \\
\hline \multicolumn{11}{|l|}{ Whites } \\
\hline SERPINA9 & rs11628722 & $\begin{array}{l}\text { nonsynonymous } \\
\text { Ala348Val }\end{array}$ & $\mathrm{G}(0.84)$ & $\mathrm{A}(0.16)$ & 1.31 & $1.00-1.70$ & 0.05 & 1.32 & $1.02-1.72$ & 0.03 \\
\hline PALLD & rs7439293 & intronic & $A(0.62)$ & $\mathrm{G}(0.38)$ & 1.24 & $1.03-1.49$ & 0.02 & 1.21 & $1.01-1.46$ & 0.04 \\
\hline IER2 & rs1042164 & $\begin{array}{l}\text { nonsynonymous } \\
\text { Val133Ala }\end{array}$ & $\mathrm{T}(0.17)$ & $\mathrm{C}(0.83)$ & 1.38 & $1.12-1.71$ & 0.003 & 1.39 & $1.12-1.72$ & 0.003 \\
\hline \multicolumn{11}{|l|}{ Blacks } \\
\hline SERPINA9 & rs11628722 & $\begin{array}{l}\text { nonsynonymous } \\
\text { Ala348Val }\end{array}$ & $\mathrm{G}(0.45)$ & $\mathrm{A}(0.55)$ & 1.26 & $1.03-1.53$ & 0.02 & 1.27 & $1.04-1.54$ & 0.02 \\
\hline EXOD1 & rs3213646 & intronic & $\mathrm{C}(0.16)$ & $\mathrm{T}(0.84)$ & 1.29 & $1.01-1.64$ & 0.04 & 1.29 & $1.01-1.65$ & 0.04 \\
\hline
\end{tabular}

\section{Results}

Race-specific proportions, means and standard deviations for the traditional risk factors are presented in table 1 . Mean values and proportions differed significantly $(\mathrm{p} \leq 0.03)$ between incident ischemic stroke cases and noncases for all risk factors.

The results from the Cox proportional hazards models, adjusting for age and gender, for all 51 SNPs are detailed in Appendix 1 (online suppl. Appendix, www.karger.com/doi/10.1159/000155637). Results are not reported for three SNPs in Whites and four SNPs in Blacks because the genotype frequencies deviated from
Hardy-Weinberg equilibrium expectations. Three SNPs in Whites (in SERPINA9, PALLD and IER2) and two SNPs in Blacks (in SERPINA9 and EXOD1) were associated ( $\mathrm{p} \leq 0.05$ ) with ischemic stroke, after adjusting for age and gender, and had the same risk allele as specified by the antecedent studies (table 2, model 1). One additional SNP in EIF2AK2 was associated with ischemic stroke in Whites, but the risk allele in the ARIC study differed from the risk allele identified in the antecedent CHD studies. The rs11628722 polymorphism in SERPINA9 was associated with incident ischemic stroke in both ethnicities [Whites: hazard rate ratio $(\mathrm{HRR})=1.31,95 \%$ CI 1.00-1.70; Blacks: HRR $=1.26,95 \%$ CI 1.03-1.53]. 
For the four SNPs that were associated in either ethnic group, traditional cardiovascular risk factors were included in the Cox proportional hazards models to evaluate possible confounding. The observed hazard ratios were essentially unchanged with addition of these risk factors to the prediction models (table 2, model 2).

\section{Discussion}

This study investigated whether 51 putative functional SNPs associated with CHD in multiple antecedent studies predict ischemic stroke among White and Black individuals from the large prospective ARIC study. The motivation for this study comes from the fact that ischemic stroke and CHD may share genetic factors. Three SNPs in Whites and two SNPs in Blacks were associated with incident ischemic stroke, even after taking into account established risk factors. The rs 11628722 polymorphism in SERPINA9 was associated with ischemic stroke in both Whites and Blacks from the ARIC study.

Few of the 51 SNPs that had previously been associated with $\mathrm{CHD}$ were associated with incident stroke in the ARIC study, and none of them would have been significantly associated with stroke after a simple Bonferroni multiple testing correction for the 51 SNPs tested. These results may be due to the fact that SNPs that confer only modest risk may not replicate in every study, or ischemic stroke and CHD may not have these genetic factors in common. However, it is noteworthy that the association between SERPINA9 and stroke was observed in both Whites and Blacks in this study. This SNP has been associated with myocardial infarction in two case-control studies and this study shows an association with stroke in both Whites and Blacks from the ARIC study. SERPINA9 is a member of clade A of the large superfamily of serine peptidase inhibitors known as serpins. Serpins are protease inhibitors that use a conformational change to inhibit target enzymes, and are involved in many cellular processes, such as coagulation, fibrinolysis, complement fixation, matrix remodeling and apoptosis [15]. Little is known about the expression of SERPINA9; however, a recent study indicated that SERPINA9 was significantly upregulated in the hippocampal tissues from Alzheimer's disease transgenic mice versus age-matched controls [16]. This study suggests that SERPINA9 may also be expressed in the human brain, consistent with our observation of an association between polymorphic variation in this gene and ischemic stroke.

SNPs Associated with CHD Predict Stroke Risk
In addition to SERPINA9, polymorphisms in palladin (PALLD) and immediate early response 2 (IER2) were associated with ischemic stroke in Whites and a polymorphism in exonuclease domain containing 1 (EXOD1) was associated with ischemic stroke in Blacks. PALLD encodes a component of the cytoskeleton that controls cell shape and motility. Vascular remodeling may lead to atherosclerosis, and the shape and cytoskeletal organization of endothelial cells is an important part of this process. Mechanical stress and strain also plays a role in atherosclerotic vascular remodeling and immediate early response genes have been shown to mediate the mechanical stress-induced pathological process in the blood vessel [17]. Although little is known about EXOD1, exonucleases have been shown to play a role in both myocardial infarction and stroke. Given their functional roles, PALLD, IER2 and EXOD1 potentially play a role in the atherosclerotic pathway. Additionally, PALLD, IER2 and EXOD1 are all expressed in the heart and brain (www. genecards.org).

A strength of this study is the prospective cohort design which is 'valuable for critically examining the potential risk factors that are initially identified through other approaches, including case-control studies' [18]. The large sample size allows for the assessment of exposures (e.g. genetic factors) of modest effect. All analyses for this study were performed separately in Whites and Blacks; however, it is important to note that ethnicity in the ARIC study is self-reported and underlying population stratification may be a potential limitation.

In summary, we have identified genes associated with incident ischemic stroke in the ARIC study. In particular, SERPINA9 was associated with stroke in both Whites and Blacks and this association does not appear to be mediated by traditional risk factors. This study underscores the utility of large-scale genomic studies of the occurrence of stroke in humans in order to identify and localize stroke susceptibility genes.

\section{Acknowledgments}

The ARIC study is carried out as a collaborative study supported by National Heart, Lung, and Blood Institute contracts N01-HC-55015, N01-HC-55016, N01-HC-55018, N01-HC-55019, N01-HC-55020, N01-HC-55021, and N01-HC-55022. The authors thank the staff and participants of the ARIC study for their important contributions. 


\section{References}

1 Rosamond W, Flegal K, Friday G, Furie K, Go A, et al, American Heart Association Statistics Committee and Stroke Statistics Subcommittee: Heart Disease and Stroke Statistics - 2007 Update: a report from the American Heart Association Statistics Committee and Stroke Statistics Subcommittee. Circulation 2007;115:e69-e171.

2 Duvall W, Vorchheimer D: Multi-bed vascular disease and atherothrombosis: scope of the problem. J Thromb Thrombolysis 2004; 17:51-61.

-3 Wilson P, D'Agostino RB, Levy D, Belanger AM, Silbershatz H, Kannel WB: Prediction of coronary heart disease using risk factor categories. Circulation 1998;97:1837-1847.

-4 Wolf P, D’Agostino RB, Belanger AJ, Kannel WB: Probability of stroke: a risk profile from the Framingham study. Stroke 1991;22:312318.

5 Morrison A, Bare LA, Chambless LE, Ellis SG, Malloy M, Kane JP, Pankow JS, Devlin JJ, Willerson JT, Boerwinkle E: Coronary heart disease risk prediction using a genetic risk score: the Atherosclerosis Risk in Communities (ARIC) Study. Am J Epidemiol 2007;166: 28-35.

-6 Bare L, Morrison AC, Rowland CM, Shiffman D, Luke MM, Iakoubova OA, Kane JP, Malloy MJ, Ellis SG, Pankow JS, Willerson JT, Devlin JJ, Boerwinkle E: Five common gene variants identify elevated genetic risk for coronary heart disease. Genet Med 2007; 9:682-689.

7 ARIC Investigators: The Atherosclerosis Risk in Communities (ARIC) Study: design and objectives. Am J Epidemiol 1989;129: 687-702.
8 Rosamond W, Folsom AR, Chambless LE, Wang CH, McGovern PG, Howard G, Copper LS, Shahar E: Stroke incidence and survival among middle-aged adults: 9-year follow-up of the Atherosclerosis Risk in Communities (ARIC) cohort. Stroke 1999; 30:736-743.

-9 Sacks F, Pfeffer MA, Moye LA, Rouleau JL, Rutherford JD, Cole TG, Brown L, Warnica JW, Arnold JM, Wun CC, Davis BR, Braunwald E: The effect of pravastatin on coronary events after myocardial infarction in patients with average cholesterol levels. Cholesterol and Recurrent Events Trial Investigators. N Engl J Med 1996;335:1001-1009.

10 Packard C, O’Reilly DS, Caslake MJ, McMahon $\mathrm{AD}$, Ford I, Cooney J, Macphee $\mathrm{CH}$, Suckling KE, Krishna M, Wilkinson FE, Rumley A, Lowe GD: Lipoprotein-associated phospholipase $\mathrm{A}_{2}$ as an independent predictor of coronary heart disease. West of Scotland Coronary Prevention Study Group. N Engl J Med 2000;343:1148-1155.

11 Iakoubova O, Tong CH, Rowland CM, et al: Association of the Trp719Arg polymorphism in kinesin-like protein 6 with myocardial infarction and coronary heart disease in two prospective studies: The Cholesterol and Recurrent Events Study (CARE) and the West of Scotland Coronary Prevention Study (WOSCOPS). J Am Coll Cardiol 2008;51: 435-443.
12 Iakoubova O, Tong CH, Chokkalingam AP, Rowland CM, et al: Asp92Asn polymorphism in the myeloid IgA Fc receptor is associated with myocardial infarction in two disparate populations: CARE and WOSCOPS. Arterioscler Thromb Vasc Biol 2006;26:2763-2768.

13 Iannone M, Taylor JD, Chen J, Li MS, Rivers P, Slentz-Kesler KA, Weiner MP: Multiplexed single nucleotide polymorphism genotyping by oligonucleotide ligation and flow cytometry. Cytometry 2000;39:131140.

14 Folsom A, Rasmussen ML, Chambless LE, Howard G, Cooper LS, Schmidt MI, Heiss G: Prospective associations of fasting insulin, body fat distribution, and diabetes with risk of ischemic stroke. Diabetes Care 1999;22: 1077-1083.

15 Law R, Zhang Q, McGowan S, Buckle AM, Silverman GA, Wong W, Rosado CJ, LangendorfCG, Pike RN, Bird PI, Whisstock JC: An overview of the serpin superfamily. Genome Biol 2006;7:216.

16 Jee S, Cho JS, Kim CK, Hwang DY, Shim SB, Lee SH, Sin JS, Park JH, Kim YS, Choi SY, Kim YK: Oligonucleotide-based analysis of differentially expressed genes in hippocampus of transgenic mice expressing NSE-controlled APPsw. Neurochem Res 2006;31: 1035-1044.

17 Liu S: Biomechanical basis of vascular tissue engineering. Crit Rev Biomed Eng 1999;27: 75-148.

18 Manolio T, Bailey-Wilson J, Collins F: Genes, environment and the value of prospective cohort studies. Nat Rev Genet 2006;7:812820 . 